\title{
Observation and identification of mechanical systems via second order sliding modes
}

\author{
J. DAVILA $\dagger$, L. FRIDMAN*† and A. POZNYAK \\ $\dagger$ Department of Control, Division of Electrical Engineering, Facultad de Ingenieria, \\ Universidad Nacional Autonoma de Mexico, Ciudad Universitaria, 04510, Mexico, D.F., Mexico \\ †Seccion de Control Automatico, CINVESTAV-IPN, AP14-740, Mexico, D.F., 07000, Mexico
}

(Received 24 November 2005; in final form 31 May 2006)

\begin{abstract}
A second-order sliding-mode observer based on the modified super-twisting algorithm providing finite time exact observation is applied for system identification. The value of the equivalent output injection is used to identify perturbations directly. Continuous time versions of least square and forgetting factor methods are proposed to identify unknown time-invariant parameters respectively.
\end{abstract}

\section{Introduction}

\subsection{Antecedents and motivations}

Modern identification theory (Ljung 1979, Eykhoff and Parks 1990, Ljung and Gunnarsson 1990) basically deals with the problem of the efficient extraction of signal and systems dynamic properties based on available data measurements. Dynamic system identification is traditionally concerned with two issues: estimation of parameters based on direct and complete state space measurements and, state estimation (filtering) of completely known nonlinear dynamics.

Parameter identification for different classes of nonlinear systems has been extensively studied during the last three decades. Basically, the class of linear and nonlinear systems whose dynamics depends linearly on the unknown parameters was considered (see, for example, Soderstrom and Stoica (1989), Ljung and Gunnarsson (1990), Lei and Chen (1991)). A general feature of these publications, is that exact state space vector measurements are assumed to be available.

The problem of observation has been actively developed within Variable Structure Theory using the Sliding Mode approach. Sliding mode observers (see, for example, the corresponding chapters in the textbooks

*Corresponding author. Email: 1fridman@servidor.unam.mx by Edwards and Spurgeon (1998) and Utkin et al. (1999) and the recent tutorials by Barbot and Floquet (2002), Edwards et al. (2002) and Poznyak (2003) are widely used due to their attractive features:

(a) Insensitivity (more than robustness) with respect to unknown inputs.

(b) Possibilities to use the values of the equivalent output injection for the unknown inputs identification (Utkin et al. 1999, Orlov 2000).

(c) Finite time convergence to the reduced order manifold.

In Hashimoto et al. (1990), Barbot et al. (1996), Utkin et al. (1999) and Barbot and Floquet (2004) a step by step form of sliding mode observers were proposed. Such observers were based on the transformation of a given system to a block triangular observable form and the step by step estimation of each state by using of the value of the equivalent output injection. Realization of this scheme caused obligatory filtration due to the necessary discretization. On the other hand, above mentioned schemes allow formulation of extended observability conditions for the systems with unknown inputs ensuring the possibilities of observations of mechanical systems with unknown inputs.

In Levant (1998, 2003), robust exact differentiators, based on the super-twisting second-order sliding-mode control algorithm, were designed ensuring a finite time 
convergence to the values of the corresponding derivatives without filtration. A new generation of observers based on second-order sliding-mode algorithms has been recently designed and applied to some practical applications (Alvarez et al. 2000, Bartolini et al. 2003, Shtessel et al. 2003, Pisano and Usai 2004, Davila et al. 2005).

\subsection{Main contributions}

In Davila et al. (2005), a second-order sliding-mode observer based on a modification of the super-twisting algorithm is proposed for observation of mechanical systems with unknown inputs and only position available. This observer ensures the finite time convergence to the value of observed velocity without filtration. But the properties of the equivalent output injection (EOI) of this observer was not studied in Davila et al. (2005).

This paper is dedicated to use the EOI of the observer proposed in Davila et al. (2005) for identification of mechanical systems:

- It is shown that for the case when a model of the system is known external perturbations could be identified directly from the EOI.

- For the identification of time invariant parameters a continuous time version of the Least Square Method (LSM) using EOI is proposed.

- For the identification of a time varying parameters vector a continuous-time Matrix Forgetting Factor (MFF) algorithm (Poznyak and Medel 1999) is modified using the EOI.

\subsection{Structure of the paper}

In $\S 2$ the problem formulation is presented. A description of the robust exact observer (Davila et al. 2005) is given in $\S 3$. The properties of EOI are analysed in $\S 4$. Section 4.2 shows that the perturbations could be identified directly from EOI. In $\S 5.2$ a continuous time version of the LSM for identification of time invariant parameters is proposed. A continuous time modification of the MFF algorithm (Poznyak and Medel 1999) for identification of time variant parameters is suggested in $\S 5.3$. In $\S 6$ examples illustrating the proposed identification algorithms are given.

\section{Problem statement}

Consider the general model of a mechanical system

$$
M(\mathbf{q}) \ddot{\mathbf{q}}+C(\mathbf{q}, \dot{\mathbf{q}}) \dot{\mathbf{q}}+P(\dot{\mathbf{q}})+G(\mathbf{q})+\Delta(t, \mathbf{q}, \dot{\mathbf{q}})=u,
$$

where $\mathbf{q} \in \mathbb{R}^{n}$ is a vector of generalized coordinates, $M(\mathbf{q})$ is an inertia matrix, $C(\mathbf{q}, \dot{\mathbf{q}})$ is a matrix of Coriolis and centrifugal forces, $P(\dot{\mathbf{q}})$ is a Coulomb friction force, which possibly contains relay terms depending on $\dot{\mathbf{q}}$, $G(\mathbf{q})$ is a vector of gravitational forces, $\Delta(t, \mathbf{q}, \dot{\mathbf{q}})$ is an uncertainty term and $u$ is the torque produced by actuators. The control input $u$ is assumed to be given by some known feedback function. Note that $M(\mathbf{q})$ is invertible for all $\mathbf{q}$, since $M(\mathbf{q})=M^{T}(\mathbf{q})$ is strictly positive definite. Also other terms are supposed to be uncertain, but the corresponding nominal functions $M_{n}(\mathbf{q})$, $C_{n}(\mathbf{q}, \dot{\mathbf{q}}), P_{n}(\dot{\mathbf{q}}), \quad G_{n}(\mathbf{q})$ are assumed to be known. Assume the vector $\mathbf{q}$ is available and the vector $\dot{\mathbf{q}}$ must be observed.

Introducing the variables $\mathbf{x}_{1}=\mathbf{q}, \mathbf{x}_{2}=\dot{\mathbf{q}}$, and the measured output $\mathbf{y}=\mathbf{x}_{1}$ the model (1) can be rewritten in the state-space form

$$
\left.\begin{array}{rl}
\dot{\mathbf{x}}_{1} & =\mathbf{x}_{2} \\
\dot{\mathbf{x}}_{2} & =F\left(t, \mathbf{x}_{1}, \mathbf{x}_{2}, u\right)+\xi\left(t, \mathbf{x}_{1}, \mathbf{x}_{2}, u\right), \quad u=U\left(t, \mathbf{x}_{1}, \mathbf{x}_{2}\right), \\
\mathbf{y} & =\mathbf{x}_{1},
\end{array}\right\}
$$

where the nominal part of the system dynamics is represented by the function

$$
\begin{aligned}
F\left(t, \mathbf{x}_{1}, \mathbf{x}_{2}, u\right)= & -M_{n}^{-1}\left(\mathbf{x}_{1}\right)\left[C_{n}\left(\mathbf{x}_{1}, \mathbf{x}_{2}\right) \mathbf{x}_{2}\right. \\
& \left.+P_{n}\left(\mathbf{x}_{2}\right)+G_{n}\left(\mathbf{x}_{1}\right)-u\right]
\end{aligned}
$$

containing the known nominal functions $M_{n}, C_{n}, G_{n}, P_{n}$, while the uncertainties and perturbations are concentrated in the term $\xi\left(t, \mathbf{x}_{1}, \mathbf{x}_{2}, u\right)$. The solutions to system (2) are understood in Filippov's sense (Filippov 1988). It is assumed that the function $F\left(t, \mathbf{x}_{1}, \mathbf{x}_{2}, U\left(t, \mathbf{x}_{1}, \mathbf{x}_{2}\right)\right)$ and the uncertainty $\xi\left(t, \mathbf{x}_{1}, \mathbf{x}_{2}, U\left(t, \mathbf{x}_{1}, \mathbf{x}_{2}\right)\right)$ are Lebesguemeasurable and uniformly bounded in any compact region of the state-space $\left(\mathbf{x}_{1}, \mathbf{x}_{2}\right)^{T}$. This assumption means that we consider the space of "real" mechanical system variables where bounded. It will be assumed that $\mathbf{x}_{1}, \mathbf{x}_{2}, u$ are time functions. The tasks are to design an observation algorithm to obtain the value of vector $\mathbf{x}_{2}$ and an identification algorithm to get the system parameters, with only the knowledge of the state $\mathbf{x}_{1}$ and the input $u(t)$.

\section{State observation}

In this paper, for the observation and identification of mechanical systems we propose the use of the secondorder sliding-mode super-twisting based observer (Davila et al. 2005) of the form

$$
\left.\begin{array}{l}
\dot{\hat{\mathbf{x}}}_{1}=\hat{\mathbf{x}}_{2}=\alpha_{2} \lambda\left(\tilde{\mathbf{x}}_{1}\right) \operatorname{sign}\left(\tilde{\mathbf{x}}_{1}\right) \\
\dot{\hat{\mathbf{x}}}_{2}=F\left(t, \mathbf{x}_{1}, \hat{\mathbf{x}}_{2}, u\right)+\alpha_{1} \operatorname{sign}\left(\tilde{\mathbf{x}}_{1}\right) \\
\tilde{\mathbf{x}}_{1}=\mathbf{y}-\hat{\mathbf{x}}_{1}
\end{array}\right\}
$$


where $\hat{\mathbf{x}}_{1}, \hat{\mathbf{x}}_{2}$ are the estimates of the state vectors $\mathbf{x}_{1}, \mathbf{x}_{2}$ respectively; the gain matrixes $\boldsymbol{\alpha}_{1}, \boldsymbol{\alpha}_{2}$ and $\lambda\left(\hat{\mathbf{x}}_{1}\right)$ are defined as

$$
\begin{aligned}
\boldsymbol{\alpha}_{1} & =\operatorname{diag}\left\{\alpha_{1_{1}}, \alpha_{1_{2}}, \ldots, \alpha_{1_{n}}\right\}, \\
\boldsymbol{\alpha}_{2} & =\operatorname{diag}\left\{\alpha_{2_{1}}, \alpha_{2_{2}}, \ldots, \alpha_{2_{n}}\right\}, \\
\lambda\left(\tilde{\mathbf{x}}_{1}\right) & =\operatorname{diag}\left\{\left|\tilde{x}_{1_{1}}\right|^{1 / 2},\left|\tilde{x}_{1_{2}}\right|^{1 / 2}, \ldots,\left|\tilde{x}_{1_{n}}\right|^{1 / 2}\right\}, \\
\operatorname{sign}\left(\tilde{\mathbf{x}}_{1}\right) & =\left[\operatorname{sign}\left(\tilde{x}_{1_{1}}\right), \operatorname{sign}\left(\tilde{x}_{1_{2}}\right), \ldots, \operatorname{sign}\left(\tilde{x}_{1_{n}}\right)\right]^{T},
\end{aligned}
$$

where $\tilde{x}_{1_{1}}=x_{1_{i}}-\hat{x}_{1_{i}}, \quad i=1, \ldots, n$, are corresponding coordinates of vector $\mathbf{x}_{1}$, and constants $\alpha_{1 i}, \alpha_{2 i}$ are the correction factors designed for convergence of estimation error for each couple of coordinates $\left(x_{1_{i}}, x_{2_{i}}\right)$. An error equation takes the form

$$
\left.\begin{array}{l}
\dot{\tilde{\mathbf{x}}}_{1}=\tilde{\mathbf{x}}_{2}-\boldsymbol{\alpha}_{2} \lambda\left(\tilde{\mathbf{x}}_{1}\right) \operatorname{sign}\left(\tilde{\mathbf{x}}_{1}\right) \\
\dot{\tilde{\mathbf{x}}}_{2}=\bar{F}\left(t, \mathbf{x}_{1}, \mathbf{x}_{2}, \hat{\mathbf{x}}_{2}, u\right)+\xi\left(t, \mathbf{x}_{1}, \mathbf{x}_{2}, u\right)-\boldsymbol{\alpha}_{1} \operatorname{sign}\left(\tilde{\mathbf{x}}_{1}\right),
\end{array}\right\}
$$

where $\bar{F}\left(t, \mathbf{x}_{1}, \mathbf{x}_{2}, \hat{\mathbf{x}}_{2}, u\right)=F\left(t, \mathbf{x}_{1}, \mathbf{x}_{2}, u\right)-F\left(t, \mathbf{x}_{1}, \hat{\mathbf{x}}_{2}, u\right)$, $\left\|\xi\left(t, \mathbf{x}_{1}, \mathbf{x}_{2}, u\right)\right\| \leq \xi^{+}$. Let us denote $\vec{f}_{i}\left(t, \mathbf{x}_{1}, \mathbf{x}_{2}, \hat{\mathbf{x}}_{2}, u\right)$ and $\xi_{i}\left(t, \mathbf{x}_{1}, \mathbf{x}_{2}, u\right)$ to the $i$ th row of the functions vector $\bar{F}\left(t, \mathbf{x}_{1}, \mathbf{x}_{2}, \hat{\mathbf{x}}_{2}, u\right)$ and the perturbation $\xi\left(t, \mathbf{x}_{1}, \mathbf{x}_{2}, u\right)$ respectively. Due the boundedness assumption it is possible to find an upperbound for each couple of coordinates such that

$$
\left|\tilde{f}_{i}\left(t, \mathbf{x}_{1}, \mathbf{x}_{2}, \hat{\mathbf{x}}_{2}, u\right)+\xi_{i}\left(t, \mathbf{x}_{1}, \mathbf{x}_{2}, u\right)\right|<f_{i}^{+}
$$

Theorem 3.1 (Davila et al. 2005): Suppose that condition (5) holds for system (2), and the parameters of the observer (3) are selected according to

$$
\left.\begin{array}{l}
\alpha_{1_{i}}>f_{i}^{+}, \\
\alpha_{2_{i}}>\sqrt{\frac{2}{\alpha_{1_{i}}-f_{i}^{+}}} \frac{\left(\alpha_{1_{i}}+f_{i}^{+}\right)\left(1+p_{i}\right)}{\left(1-p_{i}\right)},
\end{array}\right\}
$$

where $p_{i}$ are some constants to be chosen $0<p_{i}<1$, $i=1, \ldots, n$, and $\alpha_{1}=\operatorname{diag}\left\{\alpha_{1_{1}}, \alpha_{1_{2}}, \ldots, \alpha_{1_{n}}\right\}, \alpha_{2}=\operatorname{diag}$ $\left\{\alpha_{2_{1}}, \alpha_{2_{2}}, \ldots, \alpha_{2_{n}}\right\}$. Then, the observer (3) ensures the convergence of the estimated states $(\hat{\mathbf{x}}, \dot{\hat{\mathbf{x}}})$, to the real value of the states $(\mathbf{x}, \dot{\mathbf{x}})$ after a first time transient and there exists a time constant $t_{0}$ such that for all $t \geq t_{0}$, $\left(\hat{\mathbf{x}}_{1}, \hat{\mathbf{x}}_{2}\right)=\left(\mathbf{x}_{1}, \mathbf{x}_{2}\right)$.

The proof of Theorem 3.1 is given in Davila et al. (2005).

\section{Equivalent output injection analysis}

\subsection{Equivalent output injection}

The finite time convergence to the second order sliding mode set ensures that there exists the time constant $t_{0}>0$ such that for all $t \geq t_{0}$ the following identity holds

$$
0 \equiv \dot{\tilde{\mathbf{x}}}_{2} \equiv \bar{F}\left(t, \mathbf{x}_{1}, \mathbf{x}_{2}, \hat{\mathbf{x}}_{2}, u\right)+\xi\left(t, \mathbf{x}_{1}, \mathbf{x}_{2}, u\right)-\boldsymbol{\alpha}_{1} \operatorname{sign}\left(\tilde{\mathbf{x}}_{1}\right),
$$

notice $\bar{F}\left(t, \mathbf{x}_{1}, \mathbf{x}_{2}, \hat{\mathbf{x}}_{2}, u\right)=F\left(t, \mathbf{x}_{1}, \mathbf{x}_{2}, u\right)-F\left(t, \mathbf{x}_{1}, \hat{\mathbf{x}}_{2}, u\right)=0$ because $\hat{\mathbf{x}}_{2}=\mathbf{x}_{2}$. Then the equivalent output injection $z_{e q}$ is given by

$$
z_{e q}(t) \equiv \alpha_{1} \operatorname{sign}\left(\tilde{\mathbf{x}}_{1}\right) \equiv \xi\left(t, \mathbf{x}_{1}, \mathbf{x}_{2}, u\right) .
$$

We said before the term $\xi\left(t, \mathbf{x}_{1}, \mathbf{x}_{2}, u\right)$ is composed by uncertainties and perturbations. This term could be written as

$$
\xi\left(t, \mathbf{x}_{1}, \mathbf{x}_{2}, u\right)=\zeta(t)+\Delta F\left(t, \mathbf{x}_{1}, \mathbf{x}_{2}, u\right),
$$

where $\zeta(t)$ is an external perturbation term and $\Delta F\left(t, \mathbf{x}_{1}, \mathbf{x}_{2}, u\right)$ concentrates the parameter uncertainties.

Theoretically, the equivalent output injection is the result of an infinite switching frequency of the discontinuous term $\boldsymbol{\alpha}_{1} \operatorname{sign}\left(\tilde{\mathbf{x}}_{1}\right)$. Nevertheless, the realization of the observer produces high (finite) switching frequency making necessary the application of a filter. To eliminate the high frequency component we will use the filter of the form

$$
\tau \dot{\bar{z}}_{e q}(t)=-\bar{z}_{e q}(t)+z_{e q}(t)
$$

where $\tau \in \mathbb{R}$ and $h \ll \tau \ll 1$, and where $h$ is a sampling step.

It is possible to rewrite $z_{e q}$ as result of filtering process in the following form

$$
z_{e q}(t)=\bar{z}_{e q}(t)+\varepsilon(t)
$$

where $\varepsilon(t) \in \mathbb{R}^{n}$ is the difference caused by the filtration and $\bar{z}_{e q}(t)$ is the filtered version of $z_{e q}(t)$.

Nevertheless, as it is shown in Utkin (1992) and Fridman (1999)

$$
\lim _{\substack{\tau \rightarrow 0 \\ h / \tau \rightarrow 0}} \bar{z}_{e q}(\tau, h)=z_{e q}(t)
$$

then, it is possible to assume that the equivalent output injection is equal to the output of the filter.

\subsection{Perturbation identification}

Consider the case where the nominal model is totally known, for all $t>t_{0}$ the uncertain part $\Delta F\left(t, \mathbf{x}_{1}, \mathbf{x}_{2}, u\right)=0$. The equivalent output injection takes the form

$$
\bar{z}_{e q}(t)=\alpha_{1} \operatorname{sign}\left(\tilde{\mathbf{x}}_{1}\right)=\zeta(t) .
$$

The result of the filtering process will hold

$$
\lim _{\substack{\tau \rightarrow 0 \\ h / \tau \rightarrow 0}} \bar{z}_{e q}(\tau)=\zeta(t),
$$


Then, any bounded perturbation can be identified, even in the case of discontinuous perturbations, using directly the filter output (see $\S 9.1$ ).

\section{Parameter identification}

In mechanical system observation and identification, we deal with the data set of a continuous-time nature. That's why an implementation of any standard discretization scheme is related to unavoidable losses of an existing information. This produces a systematical error basically caused by the estimation of derivatives of the considered process. As shown above, the proposed second-order sliding mode technique provides an estimation of derivatives converging in a finite time that permits to avoid an additional error arising during any standard discretization scheme implementation. Below we present the continuous-time version of LS- and FF-algorithms based on the proposed secondorder sliding mode observation scheme. Notice that the proposed algorithm can be implemented in analogue devices directly.

\subsection{Regressor form}

Let us consider the not perturbed case when $\zeta(t)=0$ and $\xi\left(t, \mathbf{x}_{1}, \mathbf{x}_{2}, u\right)=\Delta F\left(t, \mathbf{x}_{1}, \mathbf{x}_{2}, u\right)$. The systems acceleration (i.e. $\dot{\mathbf{x}}_{2}$ ) can be represented as a sum of a well-known part and an uncertain part.

$$
\dot{\mathbf{x}}_{2}=F\left(t, \mathbf{x}_{1}, \mathbf{x}_{2}, u\right)+\Delta F\left(t, \mathbf{x}_{1}, \mathbf{x}_{2}, u\right),
$$

where $F\left(t, \mathbf{x}_{1}, \mathbf{x}_{2}, u\right) \in \mathbb{R}^{n}$ is a completely known part of the system and $\Delta F\left(t, \mathbf{x}_{1}, \mathbf{x}_{2}, u\right)$ is an uncertain part. Using the regressor notation (Soderstrom and Stoica 1989) we can write the uncertain part as

$$
\Delta F\left(t, \mathbf{x}_{1}, \mathbf{x}_{2}, u\right)=\theta(t) \varphi\left(t, \mathbf{x}_{1}, \mathbf{x}_{2}, u\right),
$$

where $\theta(t) \in \mathbb{R}^{n \times l}$ is a matrix composed by the value of the uncertain parameters of the functions $M, C, G, P$ and $\varphi\left(t, \mathbf{x}_{1}, \mathbf{x}_{2}, u\right) \in \mathbb{R}^{l}$ is a known nonlinear functions vector. System (2) takes the form

$$
\left.\begin{array}{rl}
\dot{\mathbf{x}}_{1} & =\mathbf{x}_{2}, \\
\dot{\mathbf{x}}_{2} & =F\left(t, \mathbf{x}_{1}, \mathbf{x}_{2}, u\right)+\theta(t) \varphi\left(t, \mathbf{x}_{1}, \mathbf{x}_{2}, u\right), \quad u=U\left(t, \mathbf{x}_{1}, \mathbf{x}_{2}\right), \\
\mathbf{y} & =\mathbf{x}_{1},
\end{array}\right\}
$$

and the observer can be rewritten as

$$
\left.\begin{array}{l}
\dot{\hat{\mathbf{x}}}_{1}=\hat{\mathbf{x}}_{2}+\alpha_{2} \lambda\left(\tilde{\mathbf{x}}_{1}\right) \operatorname{sign}\left(\tilde{\mathbf{x}}_{1}\right) \\
\dot{\hat{\mathbf{x}}}_{2}=F\left(t, \mathbf{x}_{1}, \hat{\mathbf{x}}_{2}, u\right)+\bar{\theta}(t) \varphi\left(t, \mathbf{x}_{1}, \hat{\mathbf{x}}_{2}, u\right)+\alpha_{1} \operatorname{sign}\left(\tilde{\mathbf{x}}_{1}\right),
\end{array}\right\}
$$

where $\bar{\theta} \in \mathbb{R}^{n \times l}$ is a matrix of nominal values of the parameters matrix $\theta(t)$. The error dynamics (4), for all $t \geq t_{0}$ become

$$
\left.\begin{array}{l}
\dot{\tilde{\mathbf{x}}}_{1}=\tilde{\mathbf{x}}_{2}-\alpha_{2} \lambda\left(\tilde{\mathbf{x}}_{1}\right) \operatorname{sign}\left(\tilde{\mathbf{x}}_{1}\right) \\
\dot{\tilde{\mathbf{x}}}_{2}=(\theta(t)-\bar{\theta}(t)) \varphi\left(t, \mathbf{x}_{1}, \mathbf{x}_{2}, u\right)-\boldsymbol{\alpha}_{1} \operatorname{sign}\left(\tilde{\mathbf{x}}_{1}\right) .
\end{array}\right\}
$$

Note that parameter uncertainties are concentrated in the first part of the model $(\theta(t)-\bar{\theta}(t)) \varphi\left(t, \mathbf{x}_{1}, \mathbf{x}_{2}, u\right)$.

The task is to design an algorithm which provides parameter identification for the original system (1), when only the position $\mathbf{x}_{1}$ is measurable and the nominal model $\bar{\theta}(t) \varphi\left(t, \mathbf{x}_{1}, \mathbf{x}_{2}, u\right)$ is known.

\subsection{Time-invariant parameters identification}

Consider the case when system parameters are time invariant, i.e. $\theta(t)=\theta$. Now, the equivalent output injection can be represented in the form

$$
\bar{z}_{e q}(t)=\alpha_{1} \operatorname{sign}\left(\tilde{\mathbf{x}}_{1}\right)=(\theta-\bar{\theta}) \varphi\left(t, \mathbf{x}_{1}, \mathbf{x}_{2}, u\right) .
$$

Notice that $\boldsymbol{\alpha}_{1} \operatorname{sign}\left(\tilde{\mathbf{x}}_{1}\right)$ is a known term and the finite time convergence of observer guarantees the knowledge of all the state vectors, i.e. $\varphi\left(t, \mathbf{x}_{1}, \hat{\mathbf{x}}_{2}, u\right)=\varphi\left(t, \mathbf{x}_{1}, \mathbf{x}_{2}, u\right)$ for all $t \geq t_{0}$. Equation (14) represents a linear regression model where the vector parameters to be estimated are $(\theta-\bar{\theta})$. To obtain the real system parameters $\theta$ a linear regression algorithm could be proposed from equation (14).

The recursive LSM algorithm (see for example Soderstrom and Stoica (1989)) applied for parameter identification of dynamical systems is designed using discretization of regressor and derivative of the states in order to obtain the regressor form. Then the algorithm is applied in a discrete form. Notice that the linear regressor form in Soderstrom and Stoica (1989) could be directly obtained from (14).

Defining $\Delta_{\theta}:=\theta-\bar{\theta}$, post-multiplying (14) by $\varphi^{T}\left(t, \mathbf{x}_{1}, \mathbf{x}_{2}, u\right)$ (for short notation function $\varphi\left(t, \mathbf{x}_{1}, \mathbf{x}_{2}, u\right)$ will be called as $\varphi(t))$. Now, using the auxiliary variable $\sigma$ for integration in time, the average values of equation (14) take the form

$$
\frac{1}{t} \int_{0}^{t} \bar{z}_{e q}(\sigma) \varphi^{T}(\sigma) \mathrm{d} \sigma=\Delta_{\theta} \frac{1}{t} \int_{0}^{t} \varphi(\sigma) \varphi(\sigma)^{T} \mathrm{~d} \sigma .
$$

Therefore, the system parameters can be estimated from (15) by

$$
\widehat{\Delta_{\theta}}=\left[\int_{0}^{t} \bar{z}_{e q}(\sigma) \varphi^{T}(\sigma) \mathrm{d} \sigma\right]\left[\int_{0}^{t} \varphi(\sigma) \varphi^{T}(\sigma) \mathrm{d} \sigma\right]^{-1}
$$


where $\widehat{\Delta_{\theta}}$ is the estimation of $\Delta_{\theta}$. For any square matrix the next equalities hold

$$
\left.\begin{array}{c}
\Gamma^{-1}(t) \Gamma(t)=I \\
\Gamma^{-1}(t) \dot{\Gamma}(t)+\dot{\Gamma}^{-1}(t) \Gamma(t)=0 .
\end{array}\right\}
$$

Let us define $\Gamma(t)=\left[\int_{0}^{t} \varphi(\sigma) \varphi^{T}(\sigma) \mathrm{d} \sigma\right]^{-1}$. Using (17) we can rewrite (16) in the form

$$
\dot{\widehat{\Delta_{\theta}}}=\left[\int_{0}^{t} \bar{z}_{e q}(\sigma) \varphi^{T}(\sigma) \mathrm{d} \sigma\right] \dot{\Gamma}(t)+z_{e q}(t) \varphi^{T}(t) \Gamma(t) .
$$

Now, using equation (15) we can write

$$
\dot{\widehat{\Delta_{\theta}}}=\widehat{\Delta_{\theta}} \Gamma^{-1}(t) \dot{\Gamma}(t)+\bar{z}_{e q}(t) \varphi^{T}(t) \Gamma(t) .
$$

Equalities (17) allow us to write a dynamic expression to compute $\Delta_{\theta}$ as

$$
\dot{\widehat{\Delta_{\theta}}}=\left[-\widehat{\Delta_{\theta}} \varphi(t)+\bar{z}_{e q}(t)\right] \varphi^{T}(t) \Gamma(t)
$$

In the same way, a dynamic form to find $\Gamma(t)$ is given by

$$
\dot{\Gamma}(t)=-\Gamma(t) \varphi(t) \varphi^{T}(t) \Gamma(t)
$$

The average values of the real $z_{e q}(t)$, without filtering, satisfy the equality

$$
\int_{0}^{t} z_{e q}(\sigma) \varphi(\sigma)^{T} \mathrm{~d} \sigma=\Delta_{\theta} \int_{0}^{t} \varphi(\sigma) \varphi(\sigma)^{T} \mathrm{~d} \sigma
$$

then

$$
\Delta_{\theta}=\left[\int_{0}^{t} z_{e q}(\sigma) \varphi(\sigma)^{T} \mathrm{~d} \sigma\right] \Gamma(t) .
$$

Substituting equation (9), the real values of vector parameters $\Delta_{\theta}$ holds

$$
\Delta_{\theta}=\left[\int_{0}^{t} \bar{z}_{e q}(\sigma) \varphi(\sigma)^{T} \mathrm{~d} \sigma+\int_{0}^{t} \varepsilon(\sigma) \varphi(\sigma)^{T} \mathrm{~d} \sigma\right] \Gamma(t) .
$$

Let us assume $\bar{z}_{e q}(t)=\widehat{\Delta_{\theta}} \varphi(t)$. In this case equation (20) becomes

$$
\Delta_{\theta}=\left[\widehat{\Delta_{\theta}} \int_{0}^{t} \varphi(\sigma) \varphi(\sigma)^{T} \mathrm{~d} \sigma+\int_{0}^{t} \varepsilon(\sigma) \varphi(\sigma)^{T} \mathrm{~d} \sigma\right] \Gamma(t),
$$

which can be written as

$$
\Delta_{\theta}=\widehat{\Delta_{\theta}}+\left[\int_{0}^{t} \varepsilon(\sigma) \varphi^{T}(\sigma) \mathrm{d} \sigma\right] \Gamma(t) .
$$

From equation (21) it is possible to define the convergence conditions

$$
\begin{aligned}
\sup \|t \Gamma(t)\| & <\infty, \\
\left\|\frac{1}{t} \int_{0}^{t} \varepsilon(\sigma) \varphi^{T}(\sigma) \mathrm{d} \sigma\right\| & \rightarrow 0 \quad \text { as } t \rightarrow \infty .
\end{aligned}
$$

Condition (22), known as persistent excitation condition (see for example, Soderstrom and Stoica (1989)), requires the non-singularity of the matrix $\Gamma^{-1}(t)=\int_{0}^{t} \varphi(\sigma) \varphi^{T}(\sigma) \mathrm{d} \sigma$. To avoid this restriction let us introduce the term $\rho I$ where $0<\rho \ll 1$ and $I$ is the unitary matrix and redefine $\Gamma^{-1}(t)$ as

$$
\Gamma^{-1}(t)=\int_{0}^{t}\left(\varphi(\sigma) \varphi(\sigma)^{T} \mathrm{~d} \sigma\right)+\rho I .
$$

In this case the value of $\Gamma^{-1}(t)$ is always non-singular.

Notice that the introduction of the term $\rho I$ is equivalent to set the initial conditions of (19), as

$$
\Gamma(0)=\rho^{-1} I, \quad 0<\rho \text {-small enough } .
$$

The introduction of the term $\rho$ ensures the condition $\sup \left\|t \Gamma_{t}\right\|<\infty$ but does not guarantees the convergence of the estimated parameters to the real values. The convergence of the estimated values to the real ones is ensured by the persistent excitation condition (see for example Soderstrom and Stoica (1989))

$$
\lim \inf _{t \rightarrow \infty} \frac{1}{t} \int_{0}^{t}\left(\varphi(\sigma) \varphi(\sigma)^{T} \mathrm{~d} \sigma\right)>0
$$

Condition (23) refers to the filtering process, and it gives the convergence quality of the identification. As fast as term $(1 / t) \int_{0}^{t} \varepsilon(\sigma) \varphi(\sigma)^{T} \mathrm{~d} \sigma$ converges to zero, the estimated parameters will tend to the real parameters values. The above can be summarized in Theorem 1 .

Theorem 1: The algorithms (18) and (19) ensure the convergences of $\widehat{\Delta_{\theta}} \rightarrow \Delta_{\theta}$ under conditions (22) and (23).

Remark 1: The effect of noise sensitivity of the suggested procedure can be easily seen from (23):

$$
\frac{1}{t} \int_{0}^{t} \varepsilon(\sigma) \varphi^{T}(\sigma) \mathrm{d} \sigma \rightarrow 0, \text { when } t \rightarrow \infty
$$

There $\varepsilon(t)$ is given by (9) and includes all error effects by observation noises (if there are), error in the realization of the equivalent output injection and etc. One can see that if $\varepsilon(t)$ and $\varphi(t)$ are uncorrelated and "on average" equal to zero, i.e.,

$$
\frac{1}{t} \int_{0}^{t} \varepsilon(\sigma) \mathrm{d} \sigma \rightarrow 0, \frac{1}{t} \int_{0}^{t} \varphi(\sigma) \mathrm{d} \sigma \rightarrow 0
$$

then the noise effect vanishes. 


\subsection{Time-variant parameter identification}

Consider the case when $\theta(t) \in \mathbb{R}^{n}$ is a time-variant parameter vector. In this case a continuous version of LSM has to be designed to obtain time-invariant parameter identification. Here we will suggest a combination of LSM with matrix forgetting factor (Poznyak and Medel 1999) to ensure parameters identification. Assume there are no external perturbations acting on the system, i.e. $\xi\left(t, \mathbf{x}_{1}, \mathbf{x}_{2}, u\right)=0$. The equivalent output injection is given by

$$
\bar{z}_{e q}(t)=\boldsymbol{\alpha}_{1} \operatorname{sign}\left(\tilde{\mathbf{x}}_{1}\right)=(\theta(t)-\bar{\theta}(t)) \varphi\left(t, \mathbf{x}_{1}, \mathbf{x}_{2}, u\right) .
$$

Let us call the matrix $R=R^{T} \in \mathbb{R}^{n \times n}$ as the matrix forgetting factor, satisfying the conditions $\left|\lambda_{\min }(R)\right|>0$, and $R=\varrho<1$. Define $\vartheta(t)=\theta(t)-\bar{\theta}(t)$ as the parameter vector to identify. Multiplying both sides of (24) by $\varphi(t)^{T} R^{t-\sigma}$ and averaging in time, one has

$$
\frac{1}{t} \int_{0}^{t} \bar{z}_{e q}(\sigma) \varphi^{T}(\sigma) R^{t-\sigma} \mathrm{d} \sigma=\frac{1}{t} \int_{0}^{t} \vartheta(\sigma) \varphi(\sigma) \varphi^{T}(\sigma) R^{t-\sigma} \mathrm{d} \sigma .
$$

Assuming that $\vartheta(t)$ is a slowly variant parameter $(\|\dot{\vartheta}(t)\| \leq \delta)$ we may define its estimate in the following form

$$
\hat{\vartheta}(t):=\left(\int_{0}^{t} \bar{z}_{e q}(\sigma) \varphi^{T}(\sigma) R^{t-\sigma} \mathrm{d} \sigma\right) \Gamma(t),
$$

where

$$
\Gamma(t):=\left[\int_{0}^{t} \varphi(\sigma) \varphi^{T}(\sigma) R^{t-\sigma} \mathrm{d} \sigma\right]^{-1}
$$

Notice that $\lim _{t \rightarrow \infty}\|R\|^{t} \rightarrow 0$. Then, the forgetting factor is chosen to assign a weight to the values of $\varphi(\sigma) \varphi^{T}(\sigma)$, emphasizing the actual value and making less important the past values, ensuring that the identification algorithm and the identifying parameter changes at the same ratio.

Let us define $\Theta(t)$ and $\Gamma(t)^{-1}$ as

$$
\begin{gathered}
\Theta(t):=\int_{0}^{t} \bar{z}_{e q}(\sigma) \varphi^{T}(\sigma) R^{t-\sigma} \mathrm{d} \sigma \\
\Gamma(t)^{-1}:=\int_{0}^{t} \varphi(\sigma) \varphi^{T}(\sigma) R^{t-\sigma} \mathrm{d} \sigma+\rho I .
\end{gathered}
$$

Post-multiplying (24) by $\varphi^{T}(t)$ and introducing the matrix forgetting factor

$$
\hat{\vartheta}(t) \int_{0}^{t} \varphi(\sigma) \varphi^{T}(\sigma) R^{t-\sigma} \mathrm{d} \sigma=\int_{0}^{t} \bar{z}_{e q}(\sigma) \varphi^{T}(\sigma) R^{t-\sigma} \mathrm{d} \sigma
$$

using (26) and (27) it is possible to write

$$
\begin{gathered}
\hat{\vartheta}(t) \Gamma^{-1}(t)=\Theta(t), \\
\hat{\vartheta}(t)=\Theta(t) \Gamma(t), \\
\dot{\hat{\vartheta}}(t)=\Theta(t) \dot{\Gamma}(t)+\dot{\Theta}(t) \Gamma(t) .
\end{gathered}
$$

Equation (26) could be written in a dynamical form as

$$
\dot{\Theta}(t)=\bar{z}_{e q}(t) \varphi^{T}(t)+\int_{0}^{t} \bar{z}_{e q}(\sigma) \varphi^{T}(\sigma)\left(\frac{\mathrm{d}}{\mathrm{d} t} R^{t-\sigma}\right) \mathrm{d} \sigma .
$$

Notice that by definition

$$
\left.\begin{array}{rl}
\frac{\mathrm{d}}{\mathrm{d} t} R^{t-\sigma} & =\lim _{\Delta t \rightarrow 0} \frac{1}{\Delta t}\left[R^{t+\Delta t-\sigma}-R^{t-\sigma}\right] \\
\frac{\mathrm{d}}{\mathrm{d} t} R^{t-\sigma} & =R^{t-\sigma} \lim _{\Delta t \rightarrow 0} \frac{1}{\Delta t}\left[R^{\Delta t}-I\right] .
\end{array}\right\}
$$

Let us introduce the matrix $T$ as the modal matrix of $R$ (its columns are eigenvectors of $R$ ), such that

$$
\bar{R}=T^{-1} R T=\left[\begin{array}{ccccc}
\bar{r}_{1} & 0 & \ldots & 0 & 0 \\
0 & \bar{r}_{2} & \ldots & 0 & 0 \\
\vdots & \vdots & \ddots & \vdots & \vdots \\
0 & 0 & \ldots & \bar{r}_{n-1} & 0 \\
0 & 0 & \ldots & 0 & \bar{r}_{n}
\end{array}\right]
$$

then, equation (31) can be written as

$$
\lim _{\Delta t \rightarrow 0} \frac{1}{\Delta t} R^{\Delta t}=\lim _{\Delta t \rightarrow 0} \frac{1}{\Delta t} T T^{-1} R^{\Delta t} T T^{-1}
$$

$$
\begin{gathered}
\lim _{\Delta t \rightarrow 0} \frac{1}{\Delta t} R^{\Delta t}=\lim _{\Delta t \rightarrow 0} \frac{1}{\Delta t} T\left[\begin{array}{ccccc}
\bar{r}_{1}^{\Delta t} & 0 & \ldots & 0 & 0 \\
0 & \bar{r}_{2}^{\Delta t} & \ldots & 0 & 0 \\
\vdots & \vdots & \ddots & \vdots & \vdots \\
0 & 0 & \ldots & \bar{r}_{n-1}^{\Delta t} & 0 \\
0 & 0 & \ldots & 0 & \bar{r}_{n}^{\Delta t}
\end{array}\right] T^{-1} \\
=T\left(I+\lim _{\Delta t \rightarrow 0} \frac{1}{\Delta t}\left[\begin{array}{ccc}
\bar{r}_{1}^{\Delta t}-1 & \ldots & 0 \\
\vdots & \ddots & \vdots \\
0 & \ldots & \bar{r}_{n}^{\Delta t}-1
\end{array}\right]\right) T^{-1} \\
=T\left(I+\left[\begin{array}{ccc}
\ln \bar{r}_{1} & \cdots & 0 \\
\vdots & \ddots & \vdots \\
0 & \ldots & \ln \bar{r}_{n}
\end{array}\right]\right) T^{-1} .
\end{gathered}
$$

Using (32) it is possible to define $\ln R$ as

$$
\ln R=\lim _{\Delta t \rightarrow 0} \frac{1}{\Delta t}\left[R^{\Delta t}-I\right]=T^{-1}\left[\begin{array}{ccc}
\ln \bar{r}_{1} & \cdots & 0 \\
\vdots & \ddots & \vdots \\
0 & \cdots & \ln \bar{r}_{n}
\end{array}\right] T
$$


and in consequence, equation (31) takes the form

$$
\frac{\mathrm{d}}{\mathrm{d} t} R^{t-\sigma}=R^{t-\sigma} \ln R
$$

Substituting (33) into (30) we have

$$
\begin{aligned}
& \dot{\Theta}(t)=\bar{z}_{e q}(t) \varphi^{T}(t)+\int_{0}^{t} \bar{z}_{e q}(\sigma) \varphi^{T}(\sigma) R^{t-\sigma} \ln R \mathrm{~d} \sigma, \\
& \dot{\Theta}(t)=\bar{z}_{e q}(t) \varphi^{T}(t)+\ln R \int_{0}^{t} \bar{z}_{e q}(\sigma) \varphi^{T}(\sigma) R^{t-\sigma} \mathrm{d} \sigma \\
& \dot{\Theta}(t)=\bar{z}_{e q}(t) \varphi^{T}(t)+(\ln R) \Theta(t) .
\end{aligned}
$$

From (27) one has

$$
\begin{aligned}
& \dot{\Gamma}^{-1}(t)=\varphi(t) \varphi^{T}(t)+\int_{0}^{t} \varphi(\sigma) \varphi^{T}(\sigma)\left(\frac{\mathrm{d}}{\mathrm{d} t} R^{t-\sigma}\right) \mathrm{d} \sigma \\
& \dot{\Gamma}^{-1}(t)=\varphi(t) \varphi^{T}(t)+\ln R \int_{0}^{t} \varphi(\sigma) \varphi^{T}(\sigma) R^{t-d} \mathrm{~d} \sigma \\
& \dot{\Gamma}^{-1}(t)=\varphi(t) \varphi^{T}(t)+(\ln R) \Gamma^{-1}(t) .
\end{aligned}
$$

Using (35) and (17) we can write

$$
\begin{gathered}
\dot{\Gamma}(t) \Gamma^{-1}(t)+\Gamma(t)\left(\varphi(t) \varphi^{T}(t)+\Gamma^{-1}(t) \ln R\right)=0 \\
\dot{\Gamma}(t)=-\Gamma(t)\left(\varphi(t) \varphi^{T}(t)+\Gamma^{-1}(t) \ln R\right) \Gamma(t) \\
\dot{\Gamma}(t)=-\left(\Gamma(t) \varphi(t) \varphi^{T}(t)+\ln R\right) \Gamma(t) .
\end{gathered}
$$

If we use (34) and (36), equation (29) could be written as

$$
\begin{aligned}
\dot{\hat{\vartheta}}(t)= & -\Theta(t)\left(\Gamma(t) \varphi(t) \varphi^{T}(t)+\ln R\right) \Gamma(t) \\
& +\left(\bar{z}_{e q}(t) \varphi^{T}(t)+\Theta(t) \ln R\right) \Gamma(t) \\
\dot{\hat{\vartheta}}(t)= & -\Theta(t) \Gamma(t) \varphi(t) \varphi^{T}(t) \Gamma(t)+\bar{z}_{e q}(t) \varphi^{T}(t) \Gamma(t) .
\end{aligned}
$$

From (28) it follows

$$
\begin{aligned}
& \dot{\hat{\vartheta}}(t)=-\hat{\vartheta}(t) \varphi(t) \varphi^{T}(t) \Gamma(t)+\bar{z}_{e q}(t) \varphi^{T}(t) \Gamma(t) \\
& \dot{\hat{\vartheta}}(t)=\left(\bar{z}_{e q}(t)-\hat{\vartheta}(t) \varphi(t)\right) \varphi^{T}(t) \Gamma(t) .
\end{aligned}
$$

Theorem 2: The algorithms (36) and (37) ensure the following upper bound for the estimation error:

$$
\|\Delta \vartheta(t)\|^{2} \leq n m_{2} \frac{\left(1-\alpha^{t}\right)^{2}}{(\ln \alpha)^{2}}\left(\frac{\delta \sqrt{m_{2}}}{|\ln \alpha|}+\varepsilon^{+}\right)^{2}\|\Gamma(t)\|^{2}
$$

where $\Delta \vartheta=\hat{\vartheta}-\vartheta$, under the assumption that the next conditions holds

- $\|\dot{\vartheta}(t)\| \leq \delta$

- $\|R\|=\alpha<1$

- $\left\|\varphi(t) \varphi^{T}(t)\right\|=\varphi^{T}(t) \varphi(t)=\|\varphi(t)\|^{2} \leq m_{2}$

- In (9) $\|\varepsilon(t)\| \leq \epsilon^{+}$

Proof: Let us combine (9) into (25) and it follows that

$$
\begin{aligned}
\Delta \vartheta(t)= & {\left[\int_{0}^{t} \vartheta(\sigma) \varphi(\sigma) \varphi^{T}(\sigma) R^{t-\sigma} \mathrm{d} \sigma\right.} \\
& \left.+\int_{0}^{t} \varepsilon(\sigma) \varphi^{T}(\sigma) R^{t-\sigma} \mathrm{d} \sigma\right] \Gamma(t)-\vartheta(t)
\end{aligned}
$$

$$
\begin{aligned}
\Delta \vartheta(t)= & {\left[\int_{0}^{t}(\vartheta(t)-\vartheta(\sigma)) \varphi(\sigma) \varphi^{T}(\sigma) R^{t-\sigma} \mathrm{d} \sigma\right.} \\
& \left.+\int_{0}^{t} \varepsilon(\sigma) \varphi^{T}(\sigma) R^{t-\sigma} \mathrm{d} \sigma\right]:=M(t)=M_{1}(t)+M_{2}(t)
\end{aligned}
$$

where

$$
\begin{aligned}
& M_{1}(t):=\left[\int_{0}^{t}(\vartheta(t)-\vartheta(\sigma)) \varphi(\sigma) \varphi^{T}(\sigma) R^{t-\sigma} \mathrm{d} \sigma\right] \Gamma(t) \\
& M_{2}(t):=\left[\int_{0}^{t} \varepsilon(\sigma) \varphi^{T}(\sigma) R^{t-\sigma} \mathrm{d} \sigma\right] \Gamma(t)
\end{aligned}
$$

Notice that

$$
\begin{aligned}
\|\Delta \vartheta(t)\|^{2}= & \operatorname{tr}\left\{\Delta \vartheta(t) \Delta \vartheta^{T}(t)\right\}=\operatorname{tr}\left\{M(t) M^{T}(t)\right\} \\
& \leq n\|M(t)\|^{2} \leq n\left(\left\|M_{1}(t)\right\|+\left\|M_{2}(t)\right\|\right)^{2}
\end{aligned}
$$

We need to obtain upper bounds for $\left\|M_{1}(t)\right\|$ and $\left\|M_{2}(t)\right\|$. For the norm of $M_{1}(t)$ we have

$$
\begin{aligned}
\left\|M_{1}(t)\right\| & =\left\|\left[\int_{\sigma=0}^{t}\left(\int_{s=t}^{\sigma} \dot{\vartheta}(s) \mathrm{d} s\right) \varphi(\sigma) \varphi^{T}(\sigma) R^{t-\sigma} \mathrm{d} \sigma\right] \Gamma(t)\right\| \\
& =\left\|\left[\int_{s=0}^{t} \dot{\vartheta}(s)\left(\int_{\sigma=0}^{s} \varphi(\sigma) \varphi^{T}(\sigma) R^{t-\sigma} \mathrm{d} \sigma\right) \mathrm{d} s\right] \Gamma(t)\right\| \\
& \leq\|\Gamma(t)\| \int_{s=0}^{t}\|\dot{\vartheta}(s)\|\left(\int_{\sigma=0}^{s}\left\|\varphi(\sigma) \varphi^{T}(\sigma)\right\| R^{t-\sigma} \mathrm{d} \sigma\right) \mathrm{d} s \\
& \leq\|\Gamma(t)\| \delta m_{2} \int_{s=0}^{t} \int_{\sigma=0}^{s} \alpha^{t-\sigma} \mathrm{d} \sigma \mathrm{d} s \\
& =\delta m_{2} \int_{s=0}^{t} \alpha^{t-s} \int_{\sigma=0}^{s} \alpha^{s-\sigma} \mathrm{d} \sigma \mathrm{d} s \\
& \leq\|\Gamma(t)\| \delta m_{2} \int_{s=0}^{t} \alpha^{t-s} \frac{1-\alpha^{s}}{|\ln \alpha|} \mathrm{d} s=\frac{\delta m_{2}}{|\ln \alpha|}\left[\int_{0}^{t} \alpha^{t-s} \mathrm{~d} s-t \alpha^{t}\right] \\
\left\|M_{1}(t)\right\| & \leq\|\Gamma(t)\| \frac{\delta m_{2}}{(\ln \alpha)^{2}}\left(1-\alpha^{t}\right)
\end{aligned}
$$

The norm of $M_{2}(t)$ can be written as

$$
\left\|M_{2}(t)\right\| \leq\|\Gamma(t)\| \int_{0}^{t}\|\varepsilon(\sigma)\|\|\varphi(\sigma)\|\left\|R^{t-\sigma}\right\| \mathrm{d} \sigma
$$

Assume that $\varepsilon(t)$ and $\varphi(t)$ hold the assumptions given in Theorem 2 then the last equation becomes

$\left\|M_{2}(t)\right\| \leq\|\Gamma(t)\| \varepsilon^{+} \sqrt{m_{2}} \int_{0}^{t} \alpha^{t-\sigma} \mathrm{d} \sigma=\|\Gamma(t)\| \varepsilon^{+} \sqrt{m_{2}} \frac{1-\alpha^{t}}{|\ln \alpha|}$

Substituting (42) and (43) into (41), implies the upper bound for $\|M(t)\|$ and, finally, leads to (38).

Remark 2: The real parameter values can be easily computed from $\hat{\vartheta}(t)$ as $\theta(t)=\hat{\vartheta}(t)+\bar{\theta}(t)$. 


\section{Example}

\subsection{Perturbation identification}

Consider the mathematical model of a pendulum given by

$$
\ddot{\theta}=\frac{1}{J} u-\frac{M g L}{2 J} \sin \theta-\frac{V_{S}}{J} \dot{\theta}+v(t)
$$

where $M=1.1[\mathrm{Kg}]$ is the pendulum mass, $g=9.815\left[\mathrm{~m} / \mathrm{s}^{2}\right]$ is the gravitational force, $L=0.9[\mathrm{~m}]$ is the pendulum length, $J=M L^{2}=0.891\left[\mathrm{Kg} \mathrm{m}^{2}\right]$ is the arm inertia, $V_{S}=0.18\left[\mathrm{Kg} \mathrm{m}^{2} / \mathrm{s}\right]$ is the pendulum viscous friction coefficient, and $v(t)$ is a bounded disturbance term. Assume that the angle $\theta$ is available to measure. Introducing the variables $x_{1}=\theta, x_{2}=\dot{\theta}$ and the measured output $y=\theta$ the pendulum equation can be written in the state space form as

$$
\begin{aligned}
\dot{x}_{1} & =x_{2} \\
\dot{x}_{2} & =\frac{1}{J} u-\frac{M g L}{2 J} \sin x_{1}-\frac{V_{S}}{J} x_{2}+v(t) \\
y & =x_{1} .
\end{aligned}
$$

Suppose that all the system parameters $(M=1.1$ $\left.g=9.815, \quad L=0.9, \quad J=M L^{2}=0.891, \quad V_{S}=0.18\right) \quad$ are well-known. The super-twisting observer for this system has the form

$$
\begin{aligned}
& \dot{\hat{x}}_{1}=\hat{x}_{2}+\alpha_{2}\left|\tilde{x}_{1}\right|^{1 / 2} \operatorname{sign}\left(\tilde{x}_{1}\right) \\
& \dot{\hat{x}}_{2}=\frac{1}{J} u-\frac{M g L}{2 J} \sin x_{1}-\frac{V_{S}}{J} \hat{x}_{2}+\alpha_{1} \operatorname{sign}\left(\tilde{x}_{1}\right) \\
& \tilde{x}_{1}=y-\hat{x}_{1}
\end{aligned}
$$

the equivalent output injection in this case is given by

$$
z_{\text {eq }}=\alpha_{1} \operatorname{sign}\left(\tilde{x}_{1}\right)=v(t)
$$

using a low-pass filter with $\tau=0.02[\mathrm{~s}]$ for sinusoidal external perturbation the identification is shown in figure 1. Using a filter $\tau=0.002[\mathrm{~s}]$ the perturbation identification for a discontinuous signal is shown in figure 2 .

\subsection{Time invariant parameter identification}

Consider the model of a pendulum with Coulomb friction given by the equation

$$
\ddot{\theta}=\frac{1}{J} u-\frac{M g L}{2 J} \sin \theta-\frac{V_{S}}{J} \dot{\theta}-\frac{P_{S}}{J} \operatorname{sign}(\dot{\theta}),
$$

where $M=1.1[\mathrm{Kg}]$ is the pendulum mass, $g=9.815[\mathrm{~m} / \mathrm{s}]^{2}$ is the gravitational force, $L=0.9[\mathrm{~m}]$ is the arm length, $J=M L^{2}=0.891\left[\mathrm{Kg} \mathrm{m}^{2}\right]$ is the arm inertia, $V_{S}=0.18\left[\mathrm{Kg} \mathrm{m}^{2} / \mathrm{s}\right]$ is the viscous friction coefficient, $P_{S}=0.45\left[\mathrm{Kg} \mathrm{m}^{2} / \mathrm{s}^{2}\right]$ is the Coulomb friction coefficient. Suppose that the angle $\theta$ is available to measure. Introducing the variables $x_{1}=\theta, x_{2}=\dot{\theta}$, the state space form representation for the system becomes

$$
\begin{aligned}
\dot{x}_{1} & =x_{2} \\
\dot{x}_{2} & =\frac{1}{J} u-\frac{M g L}{2 J} \sin x_{1}-\frac{V}{J} x_{2}-\frac{P}{J} \operatorname{sign}\left(x_{2}\right), \\
y & =x_{1}
\end{aligned}
$$

where $\quad a_{1}=M g L / 2 J=5.4528, \quad a_{2}=\left(V_{S} / J\right)=0.2020$, $a_{3}=P_{S} / J=0.5051$ are the unknown parameters. Let us design the super-twisting based observer as

$$
\begin{aligned}
& \dot{\hat{x}}_{1}=\hat{x}_{2}+\alpha_{2}\left|\tilde{x}_{1}\right|^{1 / 2} \operatorname{sign}\left(\tilde{x}_{1}\right) \\
& \dot{\hat{x}}_{2}=\frac{1}{J} u-\bar{a}_{1} \sin x_{1}-\bar{a}_{2} \hat{x}_{2}-\bar{a}_{3} \operatorname{sign}\left(x_{2}\right)+\alpha_{1} \operatorname{sign}\left(\tilde{x}_{1}\right), \\
& \tilde{x}_{1}=y-\hat{x}_{1}
\end{aligned}
$$

where $\bar{a}_{1}=2, \bar{a}_{2}=\bar{a}_{3}=0.1$ are the nominal values of the unknown parameters. Let the control signal be generated by a twisting controller

$$
u=-30 \operatorname{sign}\left(\theta-\theta_{d}\right)-15 \operatorname{sign}\left(\dot{\theta}-\dot{\theta}_{d}\right),
$$

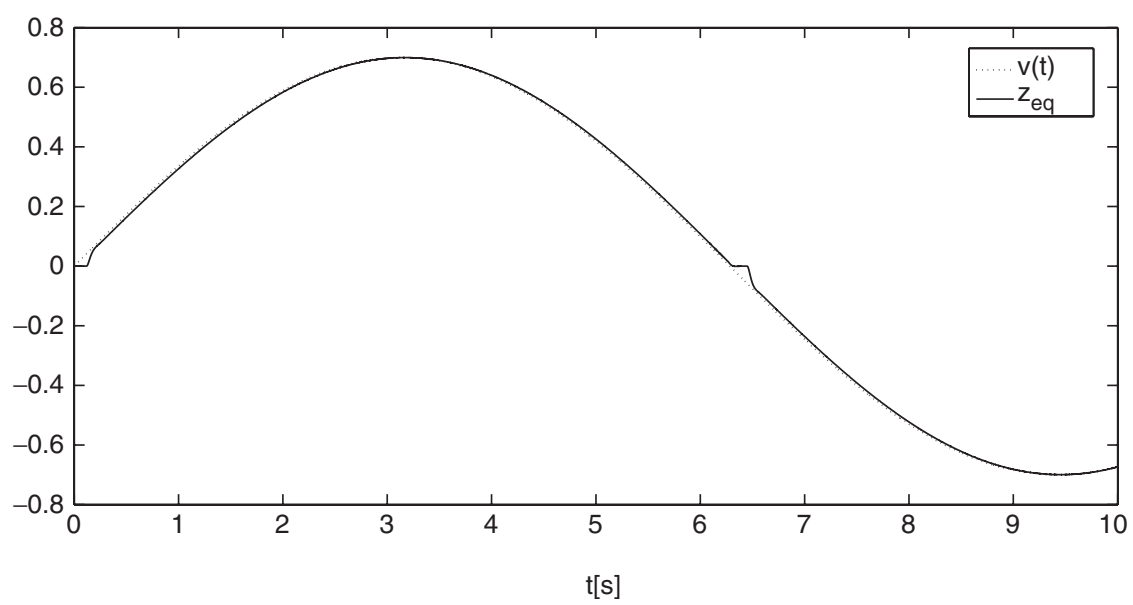

Figure 1. Sinusoidal external perturbation identification. 


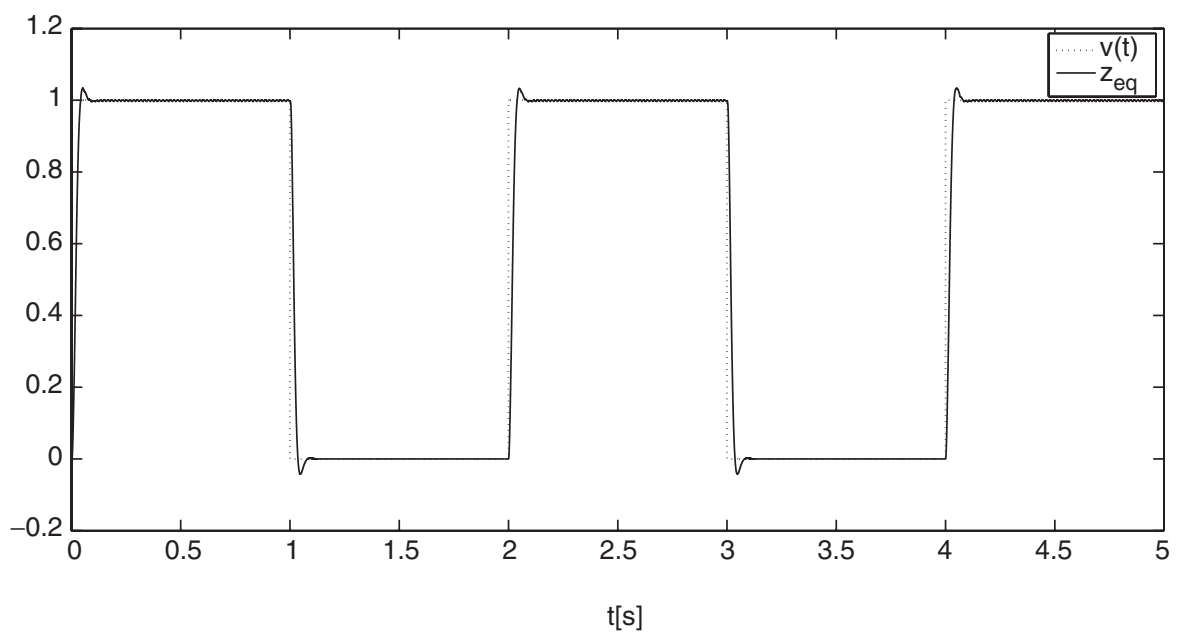

Figure 2. Discontinuous perturbation identification.
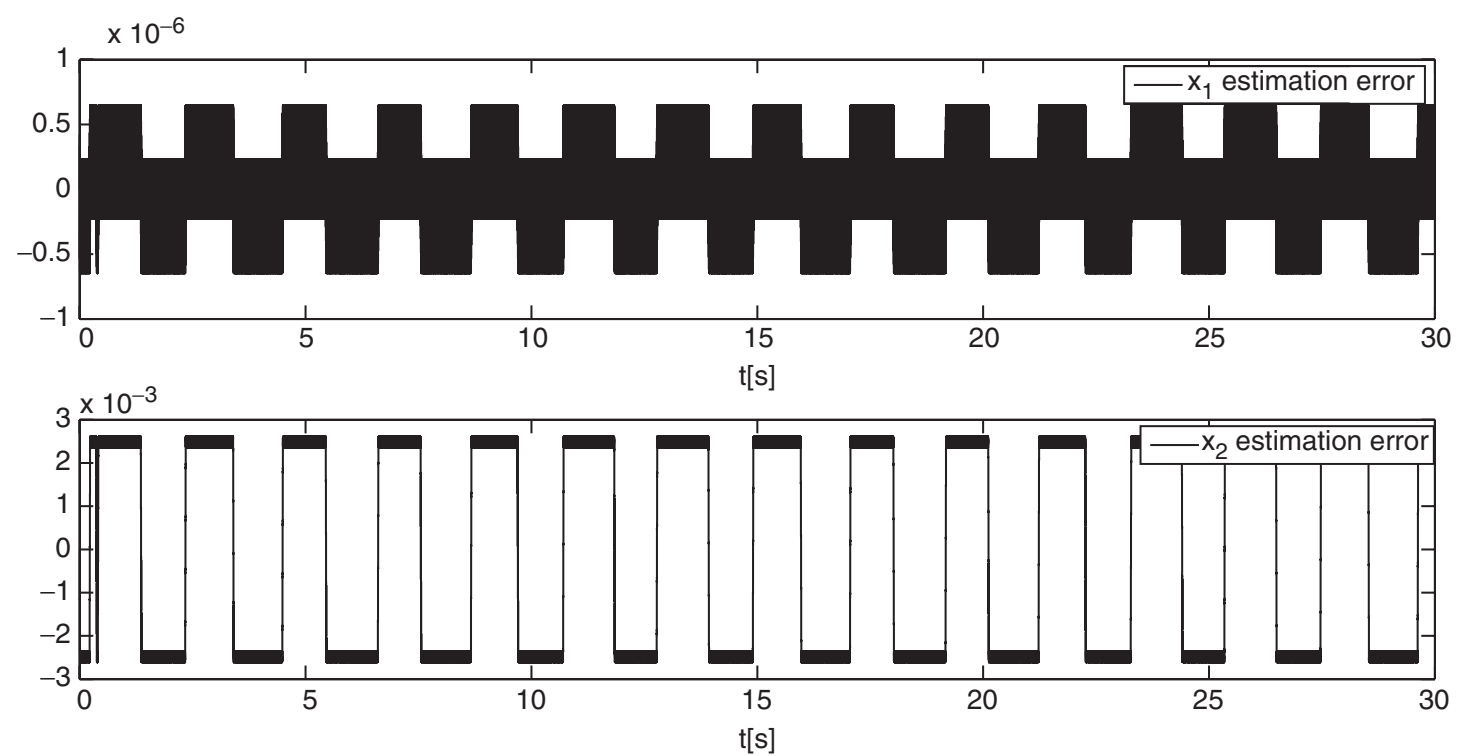

Figure 3. $x_{1}, x_{2}$ estimation error LTI case.

where the reference signal is $\theta_{d}=0.3 \sin (3 t+\pi / 4)+$ $0.3 \sin (1 / 2 t+\pi)$. For a sampling time of $\delta=0.0001$ the state estimation error is shown in figure 3 , in this case the identification variables are given by

$$
\begin{aligned}
z_{e q} & =\alpha_{1} \operatorname{sign} \tilde{x}_{1} \\
\Delta_{\theta} & =\left[\begin{array}{lll}
-a_{1}+\bar{a}_{1} & -a_{2}+\bar{a}_{2} & -a_{3}+\bar{a}_{3}
\end{array}\right] \\
\Delta_{\theta} & =\left[\begin{array}{lll}
-3.4528 & -0.1020 & -0.4051
\end{array}\right] \\
\varphi & =\left[\begin{array}{c}
\sin x_{1} \\
x_{2} \\
\operatorname{sign}\left(x_{2}\right)
\end{array}\right] .
\end{aligned}
$$

Let us apply algorithm (18). Figure 4 shows the convergence of estimated parameter to real parameter values.

\subsection{Time variant parameter identification}

Consider the model of a pendulum with time-variant damp

$$
\ddot{q}=\frac{1}{J} u-\frac{M g L}{2 J} \sin q-\frac{P_{S}}{J} \operatorname{sign}(\dot{q})-\left(0.5+0.6 \sin \frac{t}{7}\right) \dot{q},
$$



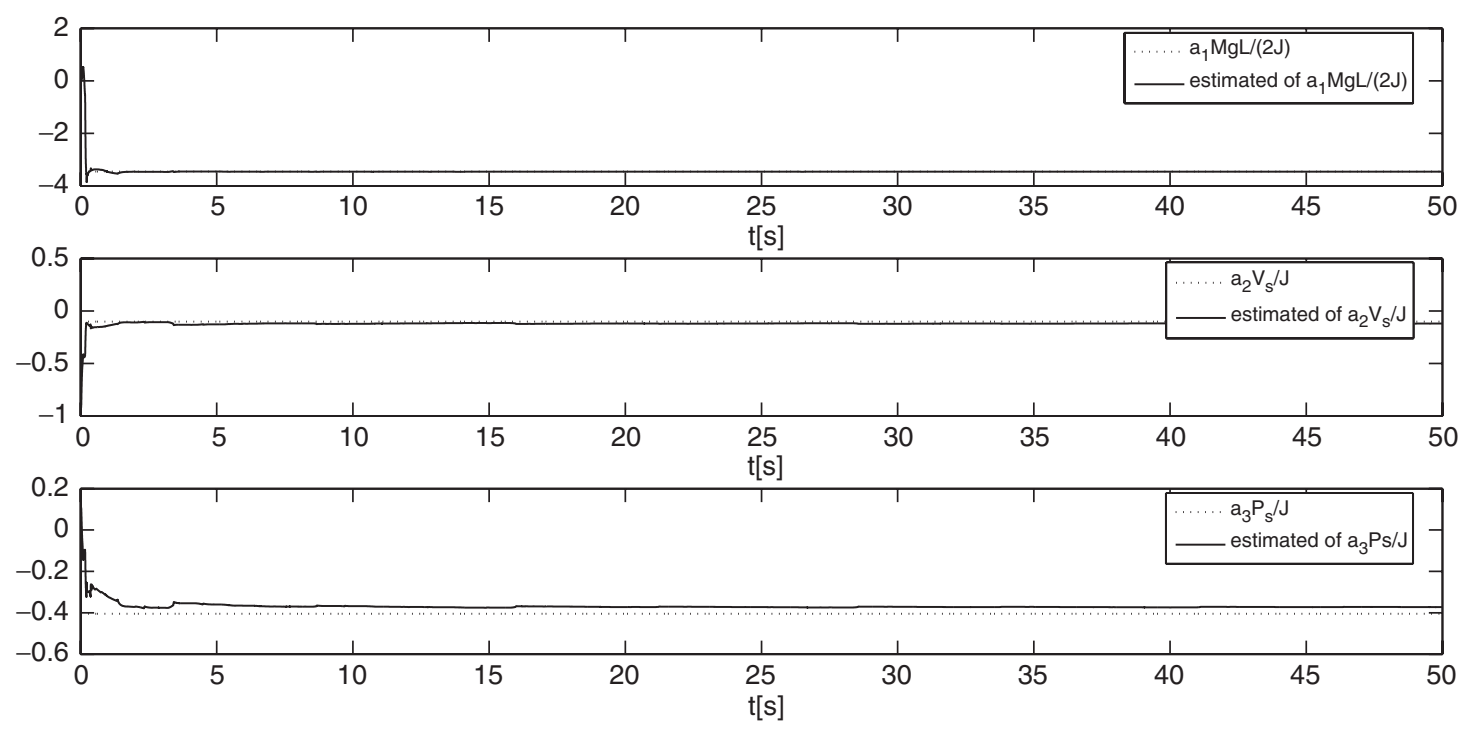

Figure 4. Parameters identification for LTI case.

where $M=1.1[\mathrm{Kg}]$ is the pendulum mass, $g=9.815\left[\mathrm{~m} / \mathrm{s}^{2}\right]$ is the gravitational force, $L=0.9[\mathrm{~m}]$ is the arm length, $J=M L^{2}=0.891\left[\mathrm{Kg} \mathrm{m}^{2}\right]$ is the pendulum inertia, $P_{s}=0.45\left[\mathrm{Kg} \mathrm{m}^{2} / \mathrm{s}^{2}\right]$ is the Coulomb friction coefficient. Notice a variable viscous damping coefficient had been introduced as $(0.5+0.6 \sin (t / 7))$. Let the system be driven by the twisting controller

$$
u=-30 \operatorname{sign}\left(\theta-\theta_{d}\right)-15 \operatorname{sign}\left(\dot{\theta}-\dot{\theta}_{d}\right),
$$

where $\theta_{d}=0.3 \sin (3 t+\pi / 4)+0.3 \sin (1 / 2 t+\pi)$ is the reference signal. Introducing the variables $x_{1}=\theta$, $x_{2}=\dot{\theta}$. System (45) can be rewritten as

$$
\begin{aligned}
\dot{x}_{1} & =x_{2} \\
\dot{x}_{2} & =\frac{1}{J} u-\frac{M g L}{2 J} \sin x_{1}-\frac{P_{S}}{J} \operatorname{sign}\left(x_{2}\right)-\left(0.5+0.6 \sin \frac{t}{7}\right) x_{2} \\
y & =x_{1} .
\end{aligned}
$$

Assuming the parameters $1 / J, M g L / 2 J, P_{S} / J$ are known. The velocity observer takes the form

$$
\begin{aligned}
& \dot{\hat{x}}_{1}=\hat{x}_{2}+\alpha_{2}\left|\tilde{x}_{1}\right|^{1 / 2} \operatorname{sign}\left(\tilde{x}_{1}\right) \\
& \dot{\hat{x}}_{2}=\frac{1}{J} u-\frac{M g L}{2 J} \sin x_{1}-\frac{P_{S}}{J} \operatorname{sign}\left(\hat{x}_{2}\right)+\alpha_{1} \operatorname{sign}\left(\tilde{x}_{1}\right) . \\
& \tilde{x}_{1}=y-\hat{x}_{1}
\end{aligned}
$$

Notice that in this case we choose $\bar{\theta}=0$. Choosing the observer parameters as $\alpha_{1}=2.2211, \alpha_{2}=19.1819$, we ensure the finite time convergence of estimated states, see figure 5. The equivalent output injection takes the form

$$
z_{e q}=\alpha_{1} \operatorname{sign} \tilde{x}_{1}=\left(0.5+0.6 \sin \frac{t}{7}\right) x_{2} .
$$

We apply the continuous time MFF method (37), (36) with

$$
\begin{aligned}
z_{e q} & =\alpha_{1} \operatorname{sign} \tilde{x}_{1}, \\
\vartheta & =-\left(0.5+0.6 \sin \frac{t}{7}\right), \\
\varphi & =x_{2}
\end{aligned}
$$

and the forgetting factor $R=0.1$. The results of time-variant parameter identification is shown in figure 6 .

\section{Conclusions}

For observation and identification of mechanical systems based on position measurements only we use the second-order sliding-mode observer ensuring the finite time convergence to zero to the velocity's observation error. This paper proposed the usage of the EOI of the above mentioned observer for identification problems:

- It is shown that the perturbations could be identified directly from the EOI.

- Continuous time version of LSM method is suggested for time invariant parameter identification.

- LSM with MFF are adapted for continuous time identification of the time invariant parameters. 

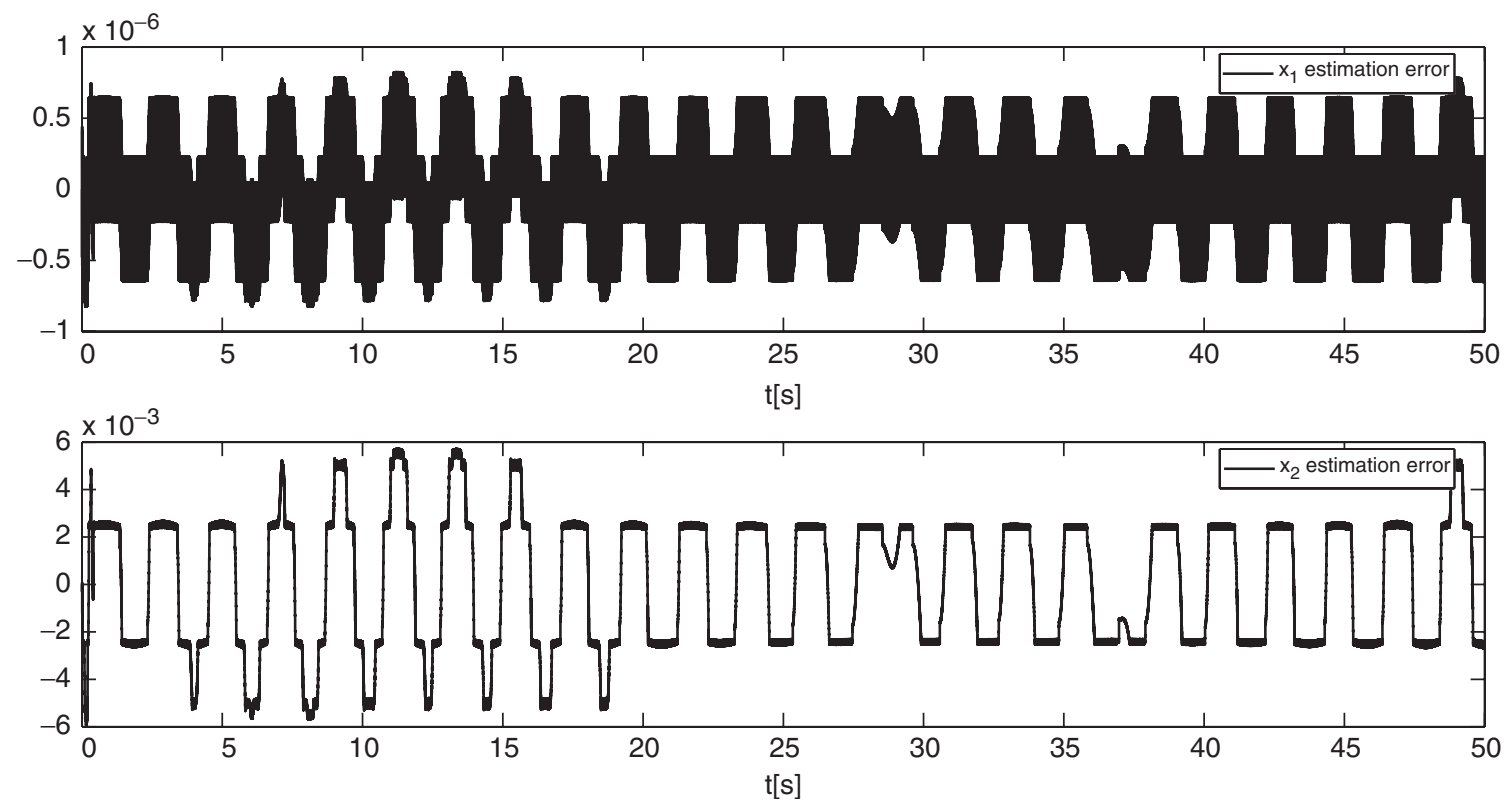

Figure 5. $x_{1}, x_{2}$ estimation error.

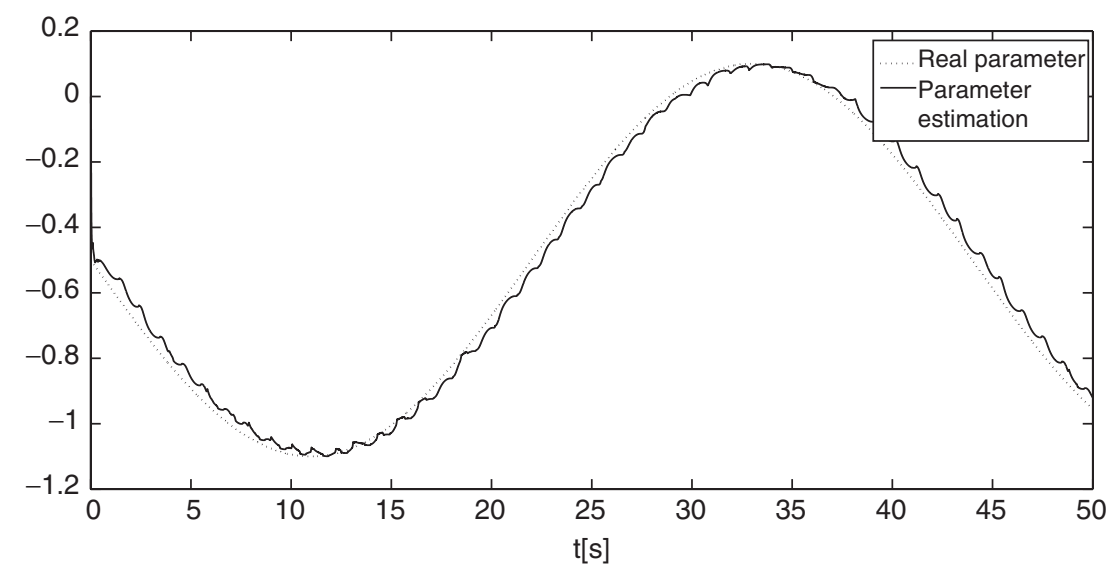

Figure 6. Parameter identification for LTV case.

\section{Acknowledgemetns}

J. Davila and L. Fridman gratefully acknowledge the financial support of this work by the Mexican CONACyT (Consejo Nacional de Ciencia y Technologia), grant no. 43807-Y, the Programa de Apoyo a Proyectos de Investigacion e Innovacion Tecnologica (PAPIIT) UNAM, grant no. 107006-2 and DGSCA-DTD

\section{References}

J. Alvarez, Y. Orlov and L. Acho, "An invariance principle for discontinuous dynamic systems with application to a Coulomb friction oscillator", J. Dyn. Sys., Meas., and Cont., 122, pp. 687-690, 2000 .
J. Barbot and T. Floquet, "A sliding mode approach of unknown input observers for linear systems", in 43th IEEE Conference on Decision and Control, Atlantis, Paradise Island, Bahamas, pp. 17241729, 2004.

J. Barbot, T. Boukhobza and M. Djemai, "Sliding mode observer for triangular input form", in 35th IEEE Conference on Decision and Control, Kobe, Japan, pp. 1489-1490, 1996.

J.P. Barbot, M. Djemai and T. Boukhobza, "Sliding mode observers" in Sliding Mode Control in Engineering, W. Perruquetti and J.P. Barbot, Eds, pp. 103-130. Control Engineering, New York: Marcel Dekker. 2002.

G. Bartolini, A. Pisano, E. Punta and E. Usai, "A survey of applications of second-order sliding mode control to mechanical systems", Inter. J. Cont., 76, pp. 875-892, 2003.

J. Davila, L. Fridman and A. Levant, "Second-order sliding-mode observer for mechanical systems", IEEE Trans. Automat. Cont., 50, pp. 1785-1789, 2005.

C. Edwards and S.K. Spurgeon, Sliding Mode Control, London: Taylor and Francis, 1998.

C. Edwards, S.K. Spurgeon and Chee Pin Tan, "On development and applications of sliding mode observers", 
in Variable Structure Systems: Towards XXIst Century, J. Xu and $\mathrm{Y}$. $\mathrm{Xu}, \mathrm{Eds}$, Lecture Notes in Control and Information Science, Berlin, Germany: Springer-Verlag, 2002, pp. 253-282.

P. Eykhoff and P.C. Parks, "Identification and system parameter estimation; where do we stand now?", Automatica, 26, pp. 3-5, 1990.

A.F. Filippov, Differential Equations with Discontinuous Right-hand Sides, Dordrecht, The Netherlands: Kluwer Academic Publishers, 1988

L. Fridman, "The problem of chattering: an averaging approach", in Variable Structure, Sliding Mode and Nonlinear Control, K.D. Young and U. Ozguner, Eds, Number 247, Lecture Notes in Control and Information Science, London: SpringerVerlag, 1999, pp. 363-386.

H. Hashimoto, V. Utkin, Jian Xin Xu, Hiroyuki Suzuki, F. Harashima, "Vss observer for linear time varying system" in Proceedings of IECON'90, Pacific Grove, CA, 1990, pp. 34-39.

G. Lei and H.F. Chen, Identification and Stochastic Adaptive Control, Boston: Birkhäuser, 1991

A. Levant, "Robust exact differentiation via sliding mode technique", Automatica, 34, pp. 379-384, 1998.

A. Levant, "High-order sliding modes: differentiation and output-feedback control", Inter. J. Cont., 76, pp. 924-941, 2003.
L. Ljung, "Asymptotic behavior of the extended kalman filter as a parameter estimator for linear systems", IEEE Trans. Automat. Cont., 24, pp. 37-50, 1979.

L. Ljung and S. Gunnarsson, "Adaptation and tracking in system identification - a survey", Automatica, 26, pp. 7-21, 1990.

Y. Orlov, "Sliding mode observer-based synthesis of state derivativefree model reference adaptive control of distributed parameter systems", ASME J. Dyn. Sys., Meas. and Cont., 122, pp. 725-731, 2000.

A. Pisano and E. Usai, "Output-feedback control of an underwater vehicle prototype by higher-order sliding modes", Automatica, 40, pp. 1525-1531, 2004.

A.S. Poznyak, "Stochastic output noise effects in sliding mode estimations", Inter. J. Cont., 76, pp. 986-999, 2003.

A.S. Poznyak and J. Medel, "Matrix forgetting factor", Int. J. Syst. Sci., 30, pp. 165-174, 1999.

Y.B. Shtessel, I.A. Shkolnikov and M.D.J. Brown, "An asymptotic second-order smooth sliding mode control", Asian J. Cont., 5, pp. 498-504, 2003.

T. Soderstrom and P. Stoica, System Identification, Cambridge, Great Britain: Prentice Hall International, 1989.

V.I. Uktin, Sliding Modes in Control and Optimization, Berlin, Germany: Springer-Verlag, 1992.

V. Utkin, J. Guldner and J. Shi, Sliding Modes in Electromechanical Systems, London: Taylor and Francis, 1999. 